\title{
Extended distance non-isocentric treatment in stereotactic body radiation therapy (SBRT) for lung cancer
}

\author{
Long Huang, Lech Papiez, Ewa Papiez, Robert Timmerman \\ Department of Radiation Oncology, University of Texas Southwestern Medical Center, Dallas, Texas, USA
}

Received September 05, 2014; Revised December 02, 2014; Accepted December 02, 2014; Published Online December 16, 2014

\section{Original Article}

\begin{abstract}
Purpose: To obtain the maximum differential non-coplanar beams angle for a faster dose dropping outside Plan Target Volume (PTV) for lung cancer treated by Stereotactic body radiation therapy (SBRT), an extended distance non-isocentric (EDNI) treatment method was explored and developed. Methods: The EDNI requires delivering of the treatment beam at $120 \mathrm{~cm}$ or farther for sauce axial distance (SAD) instead of standard $100 \mathrm{~cm}$. This change provides a more compact dose distribution around PTV and the lower toxicity to organs at risk (OAR) due to benefit of $120 \mathrm{~cm} \mathrm{SAD}$ and more choice of beam and couch angle. A hand calculation formula for the translation between 100 SAD and EDNI was used to verify the treatment plan results. A phantom for end to end study based on this EDNI technique was used to compare with standard 100 SAD deliveries for SBRT. Three patients who underwent SBRT treatment were randomly chosen to demonstrate the benefits of EDNI technique. These treatment re-plans were applied to EDNI and evaluated for conformal index (CI) of PTV, R50\% of PTV, $2 \mathrm{~cm}$ distance (D2cm) of PTV and Maximum dose $\left(D_{\max }\right)$ of OARs to compare with original clinical plans. Results: All of the cases delivered by the EDNI technique satisfied dose requirements of RTOG 0263 and showed a faster dose dropping outside of PTV than standard SAD deliveries. The distance from PTV after $1.5 \mathrm{~cm}$ for the EDNI technique had a smaller maximum dose and much lower standard deviation for dose distribution. The EDNI applied plans for patients showed less $\mathrm{R}_{50 \%}$ and $\mathrm{D}_{2 \mathrm{~cm}}$ of PTV $(\mathrm{P} \leq 0.05)$, also similar results for $\mathrm{D}_{\text {max }}$ of esophagus, trachea and spinal cord. Conclusion: The EDNI method enhances the capabilities of linear accelerators as far as the increased gradient of dose drop-off outside of PTV is concerned. More angular separation between beams leads to more compact dose distributions, which allow decreasing volume of high dose exposure in SBRT treatments and better dose distribution on sensitive organs to minimize the treatment toxicity.
\end{abstract}

Keywords: Lung Cancer; SBRT; Extend Distance; non-isocentric

\section{Introduction}

Stereotactic body radiation therapy (SBRT) has shown a promising result with local tumor control at 3 years reaching up to $98 \%$ for treatment of stage I and II of non-small cell lung cancer. ${ }^{1-3}$ SBRT plans require a more compact dose wrapping around the tumor than conventional plans. To meet this requirement, multiple non-coplanar arcs or beams avoiding opposing portals are required in SBRT plans.

The compact dose distribution for SBRT plans achieves two purposes. ${ }^{4,5}$ First, it allows much higher maximum dose inside target. Second, it allows better protecting of organs at risk (OARs) that may reside in the vicinity of the target. Due to these special characteristics of dose distribution in SBRT, additional parameters characterizing dose distribution are relevant to judge the SBRT treatment quality. ${ }^{4-6}$ Dose volume histograms (DVH) are not sufficient to appraise the value of SBRT dose distributions as indicated by parameters $\mathrm{R}_{50}$ and 2 cm distance $\left(\mathrm{D}_{2 \mathrm{~cm}}\right)$ used in the constraints imposed by protocol RTOG 0236 and subsequent SBRT protocols. ${ }^{7,8}$

One treatment parameter that can enhance the compactness of the dose distribution in SBRT, which is easily controlled during treatment planning and delivery, is the distance between the target and the radiation source. The benefit of increasing the distance between the target and the source is to decrease probability of collision between different parts of equipments (gantry and couch, gantry and patient immobilizer) and also between Linac gantry and patient body.. , 10 This results in larger spacing of angular parameters for beam directions intruding the target. This property is directly related to dose compactness as proved by earlier investigations. ${ }^{11,12}$ The increased space of angular parameters available for beam directions helps better avoidance of organs at risk in

Corresponding author: Long Huang; Department of Radiation Oncology, University of Texas Southwestern Medical Center, Dallas, Texas, USA.

Cite this article as: Huang L, Papiez L, Papiez E, Timmerman RD. Extended distance non-isocentric treatment in stereotactic body radiation therapy (SBRT) for lung cancer. Int J Cancer Ther Oncol 2015; 3(1):030112. D0I: 10.14319/ijcto.0301.12 
the body, particularly those organs at risk that dwell in the proximity of the target.

The above observations on improvement of dose distributions resulting from increasing the distance between the source and the target indicate the positive impact on SBRT with the simple remedy of increasing the distance of treatment in SBRT. This aspect of SBRT therapy has not yet been investigated. Therefore, the goal of this paper is to explore and document improvements in SBRT dosimetry for extended distance treatments while use computational and empirical tools.

In this study, we demonstrate that treatments with extended distance between the source and target have positive effects on shaping better dose distributions in SBRT. We describe the technique that is practical for extended distance treatment and applied for SBRT delivered by the linear accelerator utilized currently for radiation therapy. We named this new technique the extended distance non-isocentric (EDNI) technique.

\section{Methods and Materials}

A phantom for end to end study based on this EDVI technique was used to compare with standard 100 SAD deliveries for SBRT. Three patients who underwent SBRT treatment were randomly chosen to demonstrate the benefits of EDVI technique. These treatment re-plans were applied to EDVI and evaluated for conformal index (CI) of plan target volume (PTV), R50\% of PTV, $\mathrm{D}_{2 \mathrm{~cm}}$ of PTV and maximum dose (Dmax) of OARs to compare with original clinical plans.

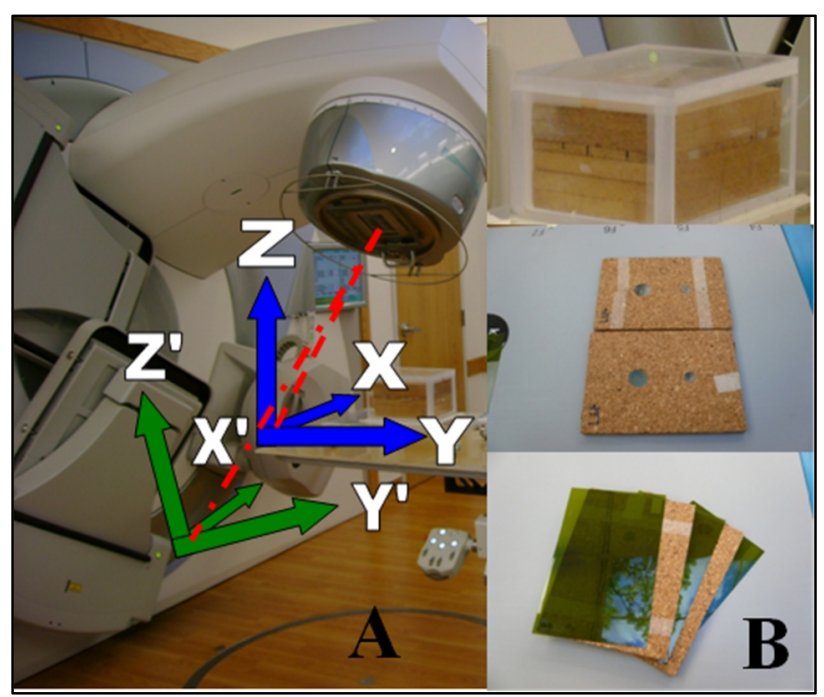

FIG. 1: (A) The conversion of coordinates for couch from SAD to EDNI technique, see detail on equation 1: (B) Lung phantoms with target $2.5 \mathrm{~cm}$ diameter, Gafchromic films inserted into each piece of corks.

\section{Lung phantom}

A cubic house-made lung phantom has an acrylic frame (dimension of $24(\mathrm{~L}) \times 17(\mathrm{~W}) \times 12(\mathrm{H}) \mathrm{cm}^{3}$, density of $\left.1.20 \mathrm{~g} / \mathrm{cm}^{3}\right)$ and was designed for our dosimetric studies. ${ }^{13}$ Cork plates (density $0.26 \mathrm{~g} / \mathrm{cm}^{3}$ ) were inserted into the frame in order to model the aerated lung tissue as shown in Figure 1B. One acrylic cylindrical target size $25 \mathrm{~mm} \times 10 \mathrm{~mm}$ is embedded in the middle of the cork plates. Targets were specifically designed to facilitate film measurement of dose at the top, middle and bottom of acrylic cylinders with Gafchromic EBT films (International Specialty Products, Wayne, NJ, USA).

Computed tomography (CT) data of the phantom in helical mode was acquired from a Philips Big Bore CT scanner (Philips Medical Systems, Cleveland, OH, USA) by using a 16 $\times 1.2 \mathrm{~mm}^{2}$ acquisition mode. For all scans, CT currents and voltage were set to $100 \mathrm{mAs}$ and $120 \mathrm{kV}$. Reconstructed slice thickness was $3 \mathrm{~mm}$. CT data were exported to a Pinnacle 9.0 (Phillips Medical Systems, Cleveland, OH, USA) treatment planning system, and the internal target volume (ITV) was contoured according to the clinical protocol used for lung SBRT. PTV was created by expanding a uniform $5 \mathrm{~mm}$ margin from ITV.

\section{Treatment planning}

We generated a sequence of three SBRT plans for each patient. Plan 1 was a standard SAD $100 \mathrm{~cm}$ plan. Ten non-coplanar beams were separated in an angular space as shown in Table 1, which can obtain compact dose distributions within the machine limit. Beams are delineated to match the target outline in each beam's eye view (BEV) and weighted to assure plan dosimetric criteria adopted in RTOG 0236. Based on Xiao et al. study ${ }^{7}$, the prescription dose is modified for $54 \mathrm{~Gy}$ for 3 fractions with heterogeneity corrections. Dose calculations were performed in Pinnacle with heterogeneity corrections utilizing the collapsed cone algorithm and dose grid size was set to $3 \mathrm{~mm}$. Pinnacle was commissioned with extended SSDs to $120 \mathrm{~cm}$.

TABLE 1: Gantry and couch angels of each beam for Plan 1, Pan 2 and Plan 3.

\begin{tabular}{ccccccc}
\hline \hline \multirow{2}{*}{$\begin{array}{c}\text { Beam } \\
\text { number }\end{array}$} & \multicolumn{2}{c}{ 100 SAD (Plan 1) } & \multicolumn{2}{c}{ 120 SAD } & (Plan 2) & \multicolumn{2}{c}{ EVDI (Plan 3) } \\
\cline { 2 - 6 } & Gantry & Couch & Gantry & Couch & Gantry & Couch \\
\hline 1 & 180 & 0 & 180 & 0 & 180 & 0 \\
2 & 220 & 345 & 220 & 345 & 220 & 335 \\
3 & 270 & 25 & 270 & 25 & 270 & 45 \\
4 & 270 & 335 & 270 & 335 & 270 & 325 \\
5 & 315 & 0 & 315 & 0 & 315 & 0 \\
6 & 30 & 270 & 30 & 270 & 40 & 270 \\
7 & 330 & 270 & 330 & 270 & 320 & 270 \\
8 & 45 & 25 & 45 & 25 & 45 & 45 \\
9 & 90 & 0 & 90 & 0 & 90 & 0 \\
10 & 150 & 0 & 150 & 0 & 150 & 0 \\
\hline \hline
\end{tabular}

Plan 2 was derived from the Plan 1 by keeping gantry and couch angles unchanged as the Plan 1 as shown in Table 1. 
The center of the target for each beam was moved along the beam's central axis to increase the distance between the source and the target center from $100 \mathrm{~cm}$ to $120 \mathrm{~cm}$. During this operation, the BEV outline of the target and corresponding MLC leaf positions were appropriately scaled for each beam. The prescriptions for the first and Plan 2 were the same, in accordance with dosimetric criteria adopted in RTOG 0236. In order to compensate for extended treatment distance, this prescription required an increase in number of monitor units assigned per each beam. Recalculation of the monitor units was provided by the planning system and verified by independent calculations.

Plan 3 was a follow up of the Plan 2, to modify the separate beams in angular degrees of freedom as much as possible, keeping an extended distance treatment of $120 \mathrm{~cm}$ between the source and the target. The angular separation in this case was clearly wider than a standard treatment distance of 100 $\mathrm{cm}$. In Table 1, we listed gantry and couch angles for the three plans. BEV apertures were changed appropriately for the new source-to-target distance and new beam directions. In Plan 3, beam weights were re-optimized so the dose coverage of the target would be identical to the dose prescribed for the first and second treatment plans.

Figure 1A shows how to convert the coordinates from SAD to EDNI for the couch that needs to be applied in order to assure the patient's body is appropriately positioned for each beam of EDNI irradiation. Equation 1 explains how to calculate this conversion.

$$
\left\{\begin{array}{l}
x \\
y \\
z
\end{array}\right\} \text { coplanar }\left\{\begin{array}{c}
x=W / 2-x_{t}-d \sin (\theta) \\
y=y_{\max }+y_{t}+d \cos (\theta) \\
z=z_{\min }-z_{t}
\end{array}\right\} \text { nocoplanar }\left\{\begin{array}{c}
x=W / 2-x_{t}-d \sin (\theta) \cos (\varphi) \\
y=y_{\max }+y_{t}+d \cos (\theta) \\
z=z_{\min }-z_{t}+d \sin (\theta) \sin (\varphi)
\end{array}\right\}
$$

In Equation 1, the coordinates (x, y, z) are presented as the couch position at $100 \mathrm{SAD}$. Gantry angle is represented by $\theta$ and couch angle is $\varphi$. Distance from isocenter is identified by $\mathrm{d}$, and width of the inside frame by $\mathrm{W}$. The largest possible positive shift of the couch down from the machine isocenter is ymax, and the largest possible shift of couch from isocenter in the direction away from gantry along the $\mathrm{z}$ axis is $\mathrm{z}$ min.

\section{Delivery and dose analysis}

The linac couch geometric accuracy is important during delivery. In monthly quality assurance, we tested the linac couch with an extension of $20 \mathrm{~cm}$ in each direction. The difference is no more than $1 \mathrm{~mm}$ on SynergyS after extending $20 \mathrm{~cm}$. Our tests indicated that the maximum difference from the extended isocenter to linac source was less than $1.7 \mathrm{~mm}$.

Due to the extension of SAD, the geometric accuracy of the couch position decreases. The geometric error is less than 2 $\mathrm{mm}$ for a $20 \mathrm{~cm}$ shift $(120 \mathrm{SAD})$ in one direction from the isocenter. Generally, the uncertainty was less than $2 \mathrm{~mm}$ for most beams as the extended distance was around 120 SAD. However, to overcome this error, we recommend that manufactures provide an independent system to monitor the couch position's accuracy.

Prior to irradiation, the beam isocenter was identified at the edge of each EBT film by using a permanent marker. This allowed us to accurately align the beam isocenter from exposed film to the plan isocenter and from the computed planar dose for dose comparison. Following irradiation, films were extracted from the phantom and scanned using Epson 10000XL (Epson America Inc., Long Beach, CA USA) flatbed scanner together with Film QA (3cognition LLC, Wayne, NJ, USA) software for analysis. Scanned films were then compared with the computed planar dose images generated by the Pinnacle planning system. Various dosimetric aspects of the computed and measured dose, including a comparison of absolute dose and relative isodose lines and gamma index (3\% $\mathrm{mm}$ ), were evaluated.

The verdict on the adequacy of PTV coverage was obtained by simple analysis of the DVH provided by treatment plans and computed from measured dose distributions. However, DVH cannot provide enough information for judging the steepness of the gradient of dose decrease away from the target. To get more information for this type of behavior of the dose cloud we create a sequence of histograms that quantify the relative change of dose between close and detached regions of the target.

After dose distributions were calculated by the treatment plans we grouped the values of doses into a histogram that provides doses from the regions defined by the PTVs. This created the first sequence of equal thickness shells to classify points outside of the target relative to their distance from the PTV. Each shell had its own dose distribution: values of dose in each shell varied between the smallest to the largest found in each shell. For each shell, the average dose, the value of maximum dose, the value of minimum dose and the value of standard deviation of as shown in Figure 3C.

\section{Results and Discussion}

\section{Dose analysis}

Plan 1 and Plan 2 had almost identical dose distributions, which were confirmed by the dose measurements performed for both cases of treatment plan irradiations. Figure 2 shows axial and coronal dose distributions in planes crossing the center of the phantom target for the three calculated plans. Plan 1 was a plan for SBRT treatment at SAD $=100 \mathrm{~cm}$ with beam directions as extensively separated as possible for treatment at SAD distance of $110 \mathrm{~cm}$, and Plan 2 used the same orientation of beams as Plan 1. However, treatment for Plan 2 was planned so that EDNI equaled $120 \mathrm{~cm}$. As Plan 3 is a modification of Plan 2, beam directions of Plan 3 were as 
extensively separated from each other as possible while avoiding collisions at treatment. The modified Plan 3 exhibited more compact dose distribution than Plans 1 and 2. Figure 2 also shows a similar comparison of measured dose distributions superimposed on calculation. Figure 2A, 2B, and $2 \mathrm{C}$ show the dose distributions of Plan 1, Plan 2 and Plan 3 treatments respectively. In bottom of Figure 2, calculated dose distributions are represented by thick lines and measures dose distributions by thin lines. Dose comparison of each plan had an excellent agreement between calculations and measurements. Based on our gamma index analysis, the passing rate of gamma index for each plan was over $95 \%$, which was similar to our clinical QA data.

\section{Shell analysis and dose compactness}

An example of shell method evaluation is shown for the Plan 1 and Plan 3 in Figure 3. The shell method provided information on how the dose distribution decreased with the distance from the PTV. This helps us in understanding how the toxicity in the organ, in which the target is located, may be affected by the irradiation of the tumor in SBRT. In Figure $3 \mathrm{~A}$, we displayed the variation of the maximum dose in each subsequent shell that was removed from the PTV by increasing distance. We noticed that the quantitative evalua- tion of dose distribution provided in Figure 3A was much more detailed than the parameter $D_{2 \mathrm{~cm}}$ that was listed originally in RTOG 0236. Figure 3A shows that up to a distance of $1.5 \mathrm{~cm}$ from PTV, the decrease in maximum dose was similar in Plan 1 and Plan 3. However, the maximum dose from the shells for Plan 3 decreased faster than Plan 1 beyond the 1.5 $\mathrm{cm}$ distances. To characterize the uniformity, or symmetry, of the dose decreases from the target it was more appropriate to look at variations of doses in each subsequent shell. Standard deviation of dose in each shell provides this information for us. We displayed the variation of standard deviation of dose in each subsequent shell removed from the PTV by increasing the distance in Figure 3B. With this graph, we can notice the curve for the standard deviation in shells for Plan 3 significantly lower than Plan 1.

Figure 3 concludes that Plan 3 for EDNI treatment was characterized by dose distributions that decreased faster away from PTV and were generally more symmetrical than Plan 1. Therefore, this dose distribution for Plan 3 better sterilized micro extensions in the vicinity of the target and caused less toxicity by exposing smaller portion of healthy tissue to the above threshold dose level in hypo-fractionated SBRT.

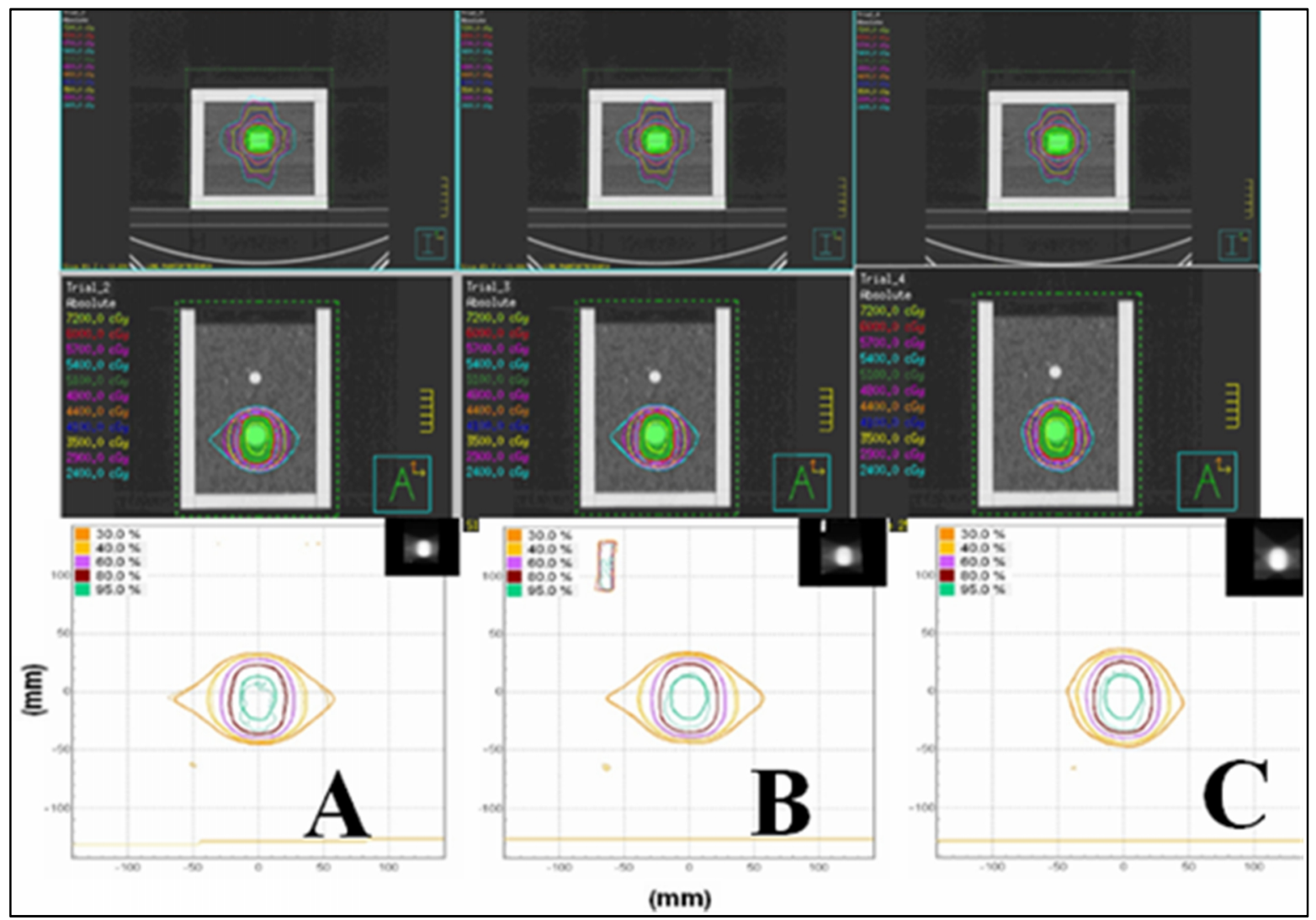

FIG. 2: Axial and coronal dose distributions for Plan 1(A), Plan 2 (B) which uses the same beam orientation as Plan 1, and Plan 3 (C), which uses an expanded set of beam orientations. The additional conformity achieved by expanding the beam orientation space is evident in the higher conformity of Plan 3. Film to calculated image matching for Plan 1 (A), film to calculated image matching for Plan 2 (B) and film to calculated image matching for Plan $3(\mathrm{C})$. 


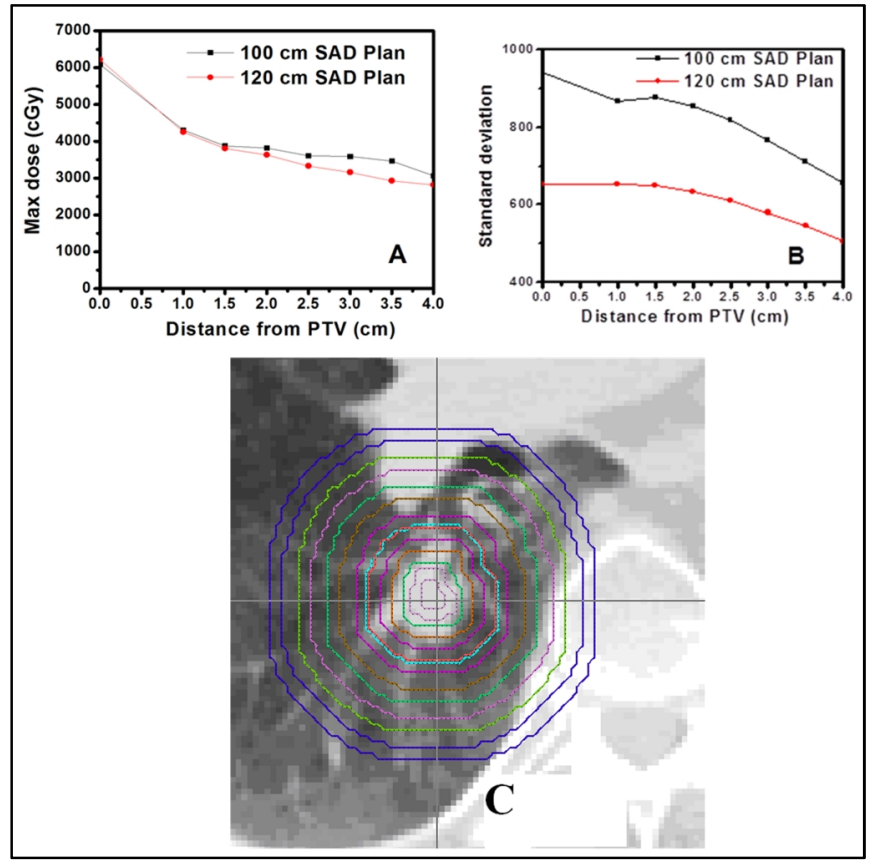

FIG. 3: (A) The max dose decreased on Plan 1 (black line) and Plan 3 (red line); (B) the standard deviation on Plan 1 (black line) and Plan 3 (red line); (C) Shells based on the distance from PTV (blue line) in the 2d representation of volumetric dose distribution.

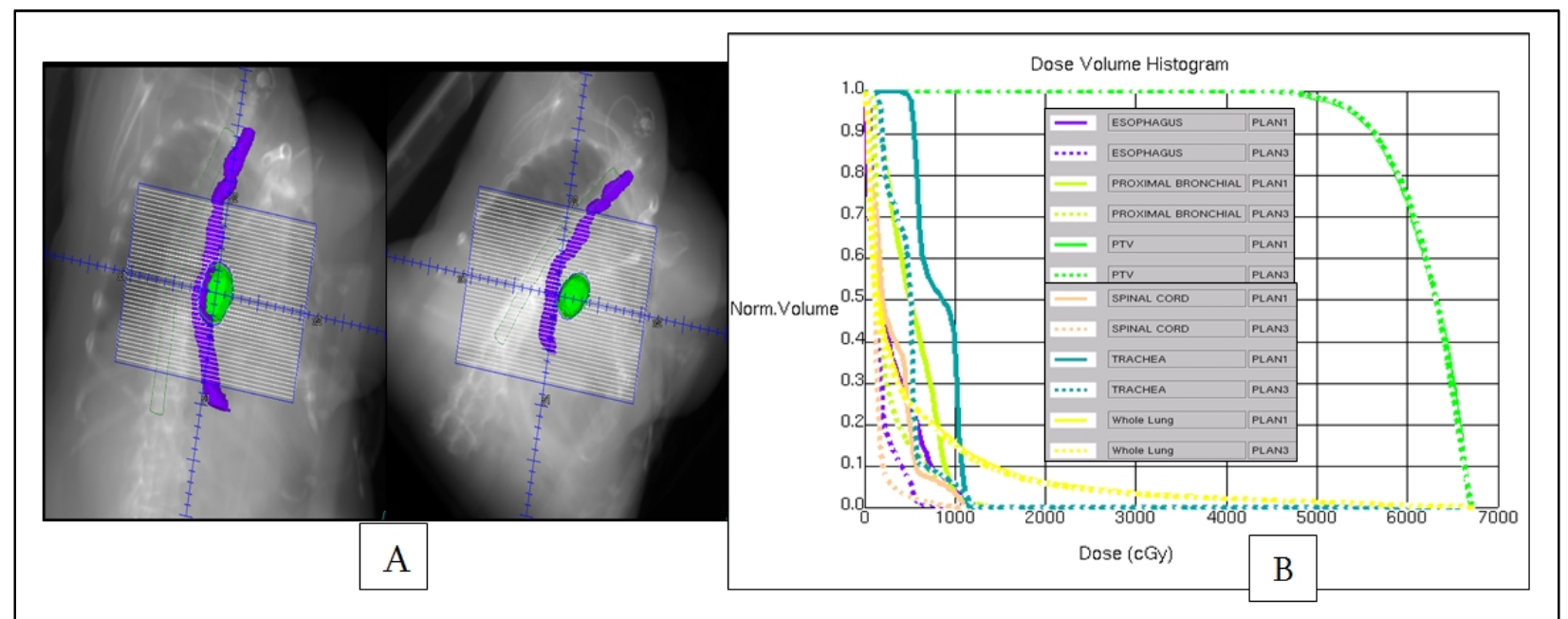

FIG. 4: (A) The BEV that esophagus cannot be avoided at Plan 1 (left), but can be avoided at Plan 3 (right): (B) The DVH of dose for Plan 1 (solid line) and Plan 3 (dash line): green for PTV, purple for esophagus, light green for proximal bronchial, orange for spinal cord, blue for trachea and yellow for whole lung.

TABLE 2: Summary of three patient cases for Plan 1 and Plan 3.

\begin{tabular}{|c|c|c|c|c|c|c|c|c|}
\hline & Plans & $\begin{array}{l}\text { PTV } \\
\text { volume } \\
(\mathrm{cm})\end{array}$ & $\begin{array}{c}\text { Ratio of Prescrip- } \\
\text { tion Isodose } \\
\text { Volume to the } \\
\text { PTV }\end{array}$ & $\begin{array}{l}\text { Ratio of } 50 \% \text { Pre- } \\
\text { scription } \\
\text { Isodose Volume to the } \\
\text { PTV, R50\% }\end{array}$ & $\begin{array}{l}\text { Maximum Dose } 2 \\
\text { cm from PTV in any } \\
\text { Direction, } D_{2 c m}(G y)\end{array}$ & $\begin{array}{l}\text { Esopha- } \\
\text { gus D } D_{\max } \\
\text { (Gy) }\end{array}$ & $\begin{array}{c}\text { Trachea } \\
D_{\max }(G y)\end{array}$ & $\begin{array}{l}\text { Spinal } \\
\text { Cord } D_{\max } \\
\text { (Gy) }\end{array}$ \\
\hline & Plan1 & 79.9 & 1.13 & 3.57 & 32.86 & 11.88 & 12.41 & 11.39 \\
\hline \multirow[t]{2}{*}{ Case 1} & Plan3 & 79.9 & 1.15 & 3.40 & 30.67 & 11.71 & 11.93 & 10.01 \\
\hline & Plan1 & 34.3 & 1.06 & 4.10 & 29.36 & 26.7 & 21.71 & 14.4 \\
\hline \multirow[t]{2}{*}{ Case 2} & Plan3 & 34.3 & 1.04 & 3.83 & 27.02 & 21.6 & 16.01 & 14.4 \\
\hline & Plan1 & 42.2 & 1.12 & 3.87 & 34.17 & 25.69 & 6.69 & 8.74 \\
\hline Case 3 & Plan3 & 42.2 & 1.10 & 3.67 & 29.15 & 20.11 & 6.26 & 8.55 \\
\hline
\end{tabular}




\section{Patient case analysis}

To apply and demonstrate the EDNI on patient cases, we analyzed three patient treatment cases of SBRT when we allowed EDNI parameters for treatment planning. Figure 4 showed SBRT lung treatment for one patient, and one of the beams needed to partially expose the organ at risk (esophagus). As is seen in Figure 4A, in Plan 1 ( $\mathrm{SAD}=100 \mathrm{~cm}$ ), it was not possible to avoid direct exposure for part of OAR while avoiding collision. However, for EDNI treatment it was possible to change the beam direction enough to avoid direct irradiation of the OAR and collision. In Figure 4B, DVH for Plan 1 and Plan 3 showed significantly dose decreasing for OARs. In comparing with Plan 1, Plan 3 definitely provided a less dose for the proximal bronchial tree, spinal cord and trachea and kept the same dose for PTV. Table 2 showed the summary of Plan 1 and Plan 3 for these three patient cases. In these three cases, the ratio of prescription isodose volume to the PTV is slightly 0.01 less in Plan 3 than Plan 1 . The ratio of $50 \%$ prescription isodose volume to the PTV ( $\left.\mathrm{R}_{50 \%}\right)$ is 0.2 lower for Plan 3. The maximum dose that is $2 \mathrm{~cm}$ from PTV in any direction $\left(\mathrm{D}_{2 \mathrm{~cm}}\right)$ is also 2 to $4 \mathrm{~Gy}$ lower for Plan 3 . We also listed several OARs such as esophagus, trachea and spinal cord for Plan 1 and Plan 3 in Table 2. All of them showed that maximum dose is lower in Plan 3.

EDNI provides a better dose distribution than the stand non-coplanar $100 \mathrm{SAD}$ delivery. However, it has own potential problems:1) a longer treatment time due to a lower dose rate for patient; 2) uncertainty increased at $120 \mathrm{~cm}$ when compared with $100 \mathrm{~cm} .3$ ). Extra work for therapists can easily lead human errors as each beam need a couch kick-out and couch shifts. A six degree robotic couch with automatic shifts software and an independent patient position monitor system with feedback can help us to reduce the treatment time and human errors

\section{Conclusion}

In this study, we explored that the EDNI method enhanced the capabilities of linear accelerators by allowing more angular separation between beams used for irradiation of the patient's body. For SBRT therapy, this capability lead to more compact dose distributions allowing decreased volume of high dose exposure in sensitive organs and minimizing the treatment toxicity. Moreover, in some cases, the technique allowed to better spare organs at risk and also possibly decreased the skin dose. We demonstrated in our investigations that the technique is easily implantable with existing commercial planning and delivery systems.

\section{Conflict of interest}

The authors declare that they have no conflicts of interest. The authors alone are responsible for the content and writing of the paper.

\section{References}

1. Zimmermann FB, Geinitz H, Schill S, et al. Stereotactic hypofractionated radiation therapy for stage I non-small cell lung cancer. Lung Cancer 2005; 48:107-14.

2. Fakiris AJ, McGarry RC, Yiannoutsos CT, et al. Stereotactic body radiation therapy for early-stage non-small-cell lung carcinoma: four-year results of a prospective phase II study. Int J Radiat Oncol Biol Phys 2009; 75:677-82.

3. McGarry RC, Papiez L, Williams M, et al. Stereotactic body radiation therapy of early-stage non-small-cell lung carcinoma: phase I study. Int $J$ Radiat Oncol Biol Phys 2005; 63:1010-5.

4. Timmerman RD, Park C, Kavanagh BD. The North American experience with stereotactic body radiation therapy in non-small cell lung cancer. $J$ Thorac Oncol 2007; 2:S101-12.

5. Timmerman RD, Kavanagh BD, Cho LC, et al. Stereotactic body radiation therapy in multiple organ sites. Journal of clinical oncology. J Clin Oncol 2007; 25:947-52.

6. Timmerman R, McGarry R, Yiannoutsos C, et al. Excessive toxicity when treating central tumors in a phase II study of stereotactic body radiation therapy for medically inoperable early-stage lung cancer. J Clin Oncol 2006; 24:4833-9.

7. Xiao Y, Papiez L, Paulus R, et al. Dosimetric evaluation of heterogeneity corrections for RTOG 0236: stereotactic body radiotherapy of inoperable stage I-II non-small-cell lung cancer. Int J Radiat Oncol Biol Phys 2009; 73:1235-42.

8. Lo SS, Fakiris AJ, Chang EL, et al. Stereotactic body radiation therapy: a novel treatment modality. Nat Rev Clin Oncol 2010; 7:44-54.

9. Hua C, Chang J, Yenice K, Chan M, Amols H. A practical approach to prevent gantry-couch collision for linac-based radiosurgery. Med Phys 2004; 31:2128-34.

10. Humm JL. Collision avoidance in computer optimized treatment planning. Med Phys 1994; 21:1053-64.

11. Webb $\mathrm{S}$. The problem of isotropically orienting $\mathrm{N}$ converging vectors in space with application to radiotherapy planning. Phys Med Biol 1995; 40:945-54.

12. Papiez L, Lu M, Langer M. On the Isotropic Distribution of Beam Directions. Math Mod Meth Appl Sci 2000; 10:991-1000.

13. Huang L, Park K, Boike T, et al. A study on the dosimetric accuracy of treatment planning for stereotactic body radiation therapy of lung cancer using average and maximum intensity projection images. Radiother Oncol 2010; 96:48-54. 